\title{
Interleukin-1 $\beta$ Expression Is Required for Lysophosphatidic Acid-Induced Lymphangiogenesis in Human Umbilical Vein Endothelial Cells
}

\author{
Chih-Hsin Lin, ${ }^{1}$ JenHer Lu, ${ }^{2}$ and Hsinyu Lee ${ }^{1,3}$ \\ ${ }^{1}$ Institute of Zoology, National Taiwan University, 1 Roosevelt Road, Section 4, Taipei 106, Taiwan \\ ${ }^{2}$ Department of Pediatrics and Pediatric Cardiology, Veterans General Hospital-Taipei, National Yang Ming University, \\ Taipei 112, Taiwan \\ ${ }^{3}$ Department of Life Science, National Taiwan University, Taipei 106, Taiwan
}

Correspondence should be addressed to JenHer Lu, jenherlu@gmail.com and Hsinyu Lee, hsinyu@ntu.edu.tw

Received 15 March 2010; Revised 24 May 2010; Accepted 28 June 2010

Academic Editor: Ricardo Saban

Copyright () 2011 Chih-Hsin Lin et al. This is an open access article distributed under the Creative Commons Attribution License, which permits unrestricted use, distribution, and reproduction in any medium, provided the original work is properly cited.

Lysophosphatidic acid (LPA) is a lipid mediator which binds to G-protein-coupled receptors and regulates various cellular responses, including inflammation of endothelial cells. Interleukin- (IL-) $1 \beta$, a proinflammatory cytokine, is elevated upon LPA treatment in human umbilical vein endothelial cells (HUVECs). Previous studies indicated that LPA upregulates vascular endothelial growth factor- (VEGF-) $\mathrm{C}$ and lymphatic marker expressions in HUVECs. However, the relationships between LPAinduced VEGF-C and IL- $1 \beta$ expressions are not clear. In this paper, we demonstrated that, in the presence of AF12198, an inhibitor of the IL-1 receptor abolished LPA-induced VEGF-C and lymphatic marker expressions in HUVECs. Furthermore, LPA-induced in vitro tube formation of HUVECs was also suppressed by pretreatment with AF12198. Our results suggest that LPA-stimulated lymphangiogenesis in HUVECs is mediated through IL- $1 \beta$-induced VEGF-C expression.

\section{Introduction}

Lysophosphatidic acid (LPA) is a low-molecular-weight lysophospholipid (LPL). Through binding to the endothelial differentiation gene (Edg) family of G-protein-coupled receptors (GPCRs), LPA regulates many cellular functions [1-3]. In serum, LPA reaches micromolar concentrations and accounts for much of the cellular growth effects of serum $[1,4]$. The major sources of LPA are mainly activated platelets, injured cells, and growth factors [5], and it can also be secreted by multiple cell types, including ovarian cancer cells [6]. LPA binds to at least five LPA receptors: $\mathrm{LPA}_{1} \sim \mathrm{LPA}_{5}$. Upon binding to these receptors, LPA regulates multiple endothelial cell functions, including proliferation, wound healing, and migration [7-13]. These results indicate that LPA plays important roles in inflammation, wound healing, and tumorigenesis [6].

Lymphangiogenesis, the process of growth and formation of new lymphatic vessels [14], occurs in normally developing tissues and pathological processes, particularly inflammation, wound healing, and cancer metastasis [15, 16]. Like angiogenesis, lymphangiogenesis is regulated by various growth factors, cytokines, and hormones [17]. Among these regulators, vascular endothelial growth factor(VEGF-) $\mathrm{C}$ is considered to be a major regulator of the lymphangiogenic process $[18,19]$. In transgenic mouse skin and mature chick chorioallantoic membrane models, VEGF-C is known to induce both lymphangiogenesis and angiogenesis $[20,21]$. Several recent studies reported that lymphatic vessels accumulate adjacent to tumor tissues highly expressing VEGF-C, and an increase in the lymphatic diameter may contribute to promotion of tumor metastasis [22]. We demonstrated that LPA stimulates VEGF$\mathrm{C}$ and lymphatic markers, including Prox-1, LYVE-1, and podoplanin expressions in human umbilical vein endothelial cells (HUVECs) [23, 24]. In addition, sphingosine-1phosphate (S1P), another bioactive lysophospholipid, was also demonstrated to induce lymphangiogenesis [25]. Those results indicate that bioactive lipids may be important lymphangiogenic regulators. 
Despite studies suggesting connections between inflammation and lymphangiogenesis, the molecular mechanisms that regulate lymphatic vessel formation remain largely unclear. Proinflammatory cytokines, such as interleukin(IL-) $1 \beta$ and tumor necrosis factor- (TNF-) $\alpha$, were shown to enhance the expression of VEGF-C by fibroblasts [26]. Moreover, IL- $1 \beta$-induced lymphangiogenesis is mediated through VEGFR-3 in the mouse cornea [27]. Those findings suggest that cytokines are important mediators of lymphangiogenesis.

In our previous studies, we demonstrated that LPA upregulates VEGF-C and lymphatic marker expressions in HUVECs [23, 24]. Moreover, we also demonstrated that LPA upregulates IL- $1 \beta$ messenger $(\mathrm{m})$ RNA expression in HUVECs [28]. In this study, we postulated that IL- $1 \beta$, the main proinflammatory cytokine, regulates LPA-induced lymphangiogenesis in HUVECs. In this report, we showed that LPA upregulated VEGF-C and lymphatic marker expressions in HUVECs in LPA1/3-, EGFR transactivation-, and IL1-dependent manners. Furthermore, we also demonstrated that LPA-induced HUVEC tube formation in vitro was suppressed by pretreatment with these inhibitors. These data demonstrate for the first time that IL- $1 \beta$ may act as an essential mediator for LPA-induced lymphangiogenesis in HUVECs.

\section{Material and Methods}

2.1. Reagents and Antibodies. Medium 199 and fetal bovine serum (FBS) were purchased from HyClone (Logan, UT), and endothelial growth medium (EGM) was purchased from Cell Application (San Diego, CA). Trypsin-EDTA was purchased from Gibco BRL (Grand Island, NY). Collagenase I, gelatin, LPA, fatty acid-free bovine serum albumin (fafBSA), and Ki16425 were purchased from Sigma-Aldrich (St. Louis, MO). Normal mouse and goat immunoglobulin Gs (IgGs) were purchased from Santa Cruz Biotechnology (Santa Cruz, CA). AG1478 and GM6001 were purchased from Calbiochem (La Jolla, CA). Penicillin and streptomycin were purchased from Invitrogen (Carlsbad, CA). AF12198 was purchased from Tocris (Ellisville, MO).

2.2. Cell Culture. Human umbilical cords were kindly provided by National Taiwan University Hospital (Institutional Review Board approval no. 9561709146). HUVECs were isolated from fresh umbilical cords by treatment with $0.1 \%$ collagenase type I (Sigma) in cord buffer at $37^{\circ} \mathrm{C}$ for $30 \mathrm{~min}$. Then endothelial cells (ECs) were collected and centrifuged. HUVECs were cultured on 1\% gelatin-coated (Sigma) $10 \mathrm{~cm}$ plates in $60 \%$ M199 medium supplemented with $100 \mathrm{U} / \mathrm{mL}$ penicillin, $100 \mathrm{mg} / \mathrm{mL}$ streptomycin, 20\% FBS, and $20 \%$ EGM. Cells underwent one passage weekly. Cells were subcultured after trypsinization and used in experiments until passage 4 .

2.3. Determination of IL-1 $\beta$ Protein Expression by an EnzymeLinked Immunosorbent Assay (ELISA). Starved HUVECs were pretreated with an inhibitor for $1 \mathrm{~h}$, followed by LPA
$(5 \mu \mathrm{M})$ treatment for an additional $24 \mathrm{~h}$. The conditioned medium was measured by an IL-1 $\beta$ ELISA kit purchased from Cayman Chemical (Ann Arbor, MI).

2.4. Determination of VEGF-C Protein Expression by an ELISA. Starved HUVECs were pretreated with an inhibitor for $1 \mathrm{~h}$, followed by LPA $(5 \mu \mathrm{M})$ treatment for an additional $24 \mathrm{~h}$, and the conditioned medium was measured by a VEGFC ELISA kit from R and D Systems (Minneapolis, MN).

2.5. CyFlow Analysis. HUVECs were starved for $16 \mathrm{~h}$ and treated as indicated. Suspensions of $10^{6}$ cells in $200 \mu \mathrm{L}$ phosphate-buffered saline (PBS) with $0.1 \%$ faf-BSA received $2 \mu \mathrm{L}$ of a rabbit antihuman Prox- 1 antibody (Abcam, Cambridge, MA), goat antihuman LYVE-1 (R \& D Systems), or mouse antihuman podoplanin (Santa Cruz Biotechnology) and were then incubated for $1 \mathrm{~h}$ at $4^{\circ} \mathrm{C}$. Antibody-conjugated cells were washed with PBS three times and incubated with an FITC-conjugated goat anti-rabbit secondary antibody (Molecular Probes, Eugene, OR), donkey anti-goat secondary antibody (Molecular Probes), or goat anti-mouse secondary antibody (DAKO, Carpentaria, CA) for $1 \mathrm{~h}$ at $4^{\circ} \mathrm{C}$. Fluorescent signals were determined by CyFlow SL (Partec, Münster, Germany) and analyzed by WinMDI version 2.8 software.

2.6. Matrigel Tube Formation Assay. Matrigel (BD Pharmingen, San Diego, CA) at $0.3 \mathrm{~mL} /$ well was plated evenly in a $24-$ well plate, and incubated at $37^{\circ} \mathrm{C}$ for $30 \mathrm{~min}$ before seeding with HUVECs $\left(5 \times 10^{4}\right.$ cells/well $)$. Tube formation was studied for $6 \mathrm{~h}$, and photographs were taken. The original magnification was 100x. The Matrigel was fixed, blocked, permeabilized, and stained with rabbit anti-mouse Prox-1 (Abcam), followed by incubation with a goat anti-rabbit AlexaFluor-555-conjugated secondary antibody (Molecular Probes). The Matrigel was thoroughly washed and incubated with Mec13.3, a direct FITC-conjugated rat anti-mouse mAb against PECAM-1 (BD Biosciences, San Jose, CA). After a further series of washes with PBS, samples were mounted on glass slides and viewed using a Zeiss fluorescence microscope (Oberkochen, Germany).

2.7. Statistical Analysis. All experiments were performed at least three times, and the data are expressed as the mean \pm SD. An analysis of variance (ANOVA) was performed using Statview 5.0 software. $P<.05$ was considered statistically significant.

\section{Results and Discussion}

3.1. LPA-Induced Lymphatic Marker Expressions Are Mediated through EGFR Transactivation-, Matrix Metalloproteinase(MMP-), LPA1/3-, and IL-1R-Dependent Pathway in HUVECs. Our previous studies demonstrated that LPA enhances IL- $1 \beta$ mRNA expression in HUVECs [28], and LPA induces the expressions of VEGF-C and lymphatic markers in human endothelial cells [23]. In addition, IL-1 $\beta$ stimulates VEGF-C expression in HUVECs [26]. 

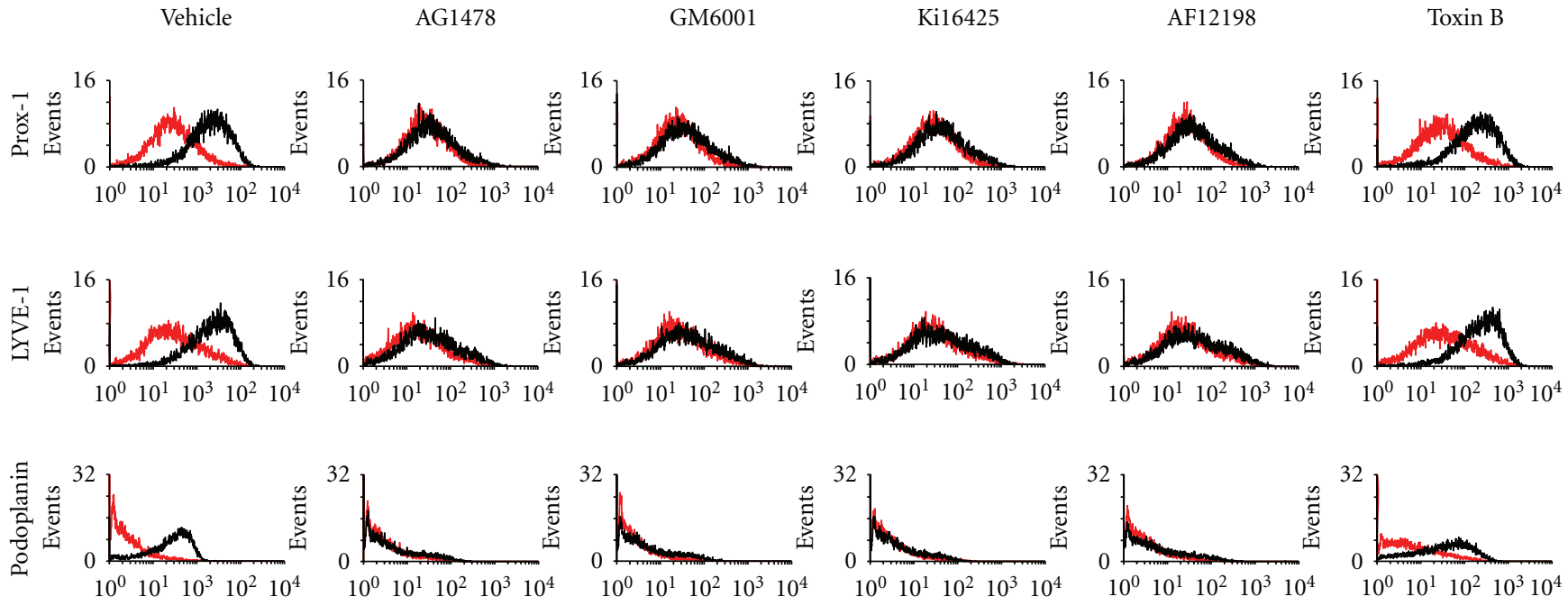

- Media

- Media

- Media

- Media

- Media

- Media

FIGURE 1: Lysophosphatidic acid- (LPA-) induced lymphatic marker expressions are mediated through endothelial growth factor receptor (EGFR) transactivation-, matrix metalloproteinase- (MMP-), LPA1/3-, and interleukin- (IL-) 1R-dependent pathways in human umbilical vein endothelial cells (HUVECs). Starved HUVECs were pretreated with AG1478 (100 nM), AF12198 (10 nM), GM6001 (10 $\mu$ M), Ki16425 $(10 \mu \mathrm{M})$, and toxin B $(10 \mathrm{nM})$ for $1 \mathrm{~h}$, followed by LPA $(5 \mu \mathrm{M})$ treatment for an additional $8 \mathrm{~h}$. HUVECs were dissociated by trypsinization and fixed with $4 \%$ paraformaldehyde. Fixed cells were labeled with antibodies for Prox-1, LYVE-1, and podoplanin, and then analyzed by CyFlow. Cells were also stained with a normal mouse or goat immunoglobulin G antibody, which served as a negative control.

Therefore, we further investigated whether IL- $1 \beta$ plays a role in LPA-induced lymphangiogenesis and whether LPA1/3 or EGFR transactivation mediates LPA-induced lymphangiogenesis in HUVECs. These enhancement effects were suppressed by pretreatment with inhibitors of LPA1/3 (Ki16425), EGFR kinase (AG1478), a broad-spectrum MMP (GM6001), and IL-1R (AF12198). However, pretreatment with a Rac inhibitor (toxin B) showed no suppressive effects (Figure 1). These results indicated that LPA's enhancement of Prox-1, LYVE-1, and podlymphaticmarker expressions are oplanin protein expressions in HUVECs is mediated by LPA1/3-, EGFR transactivation-, MMP-, and IL-1Rdependent mechanisms.

3.2. LPA-Induced IL-1 $\beta$ Protein Expression Is Mediated by EGFR Transactivation-, MMP-, LPA1/3-, and IL-1RDependent Mechanisms in HUVECs. Since LPA-induced lymphatic marker expressions are mediated by the IL-1R in HUVECs (Figure 1), we further investigated whether LPA stimulates IL- $1 \beta$ expression and whether LPA1/3 or EGFR transactivation mediates LPA-induced IL- $1 \beta$ expression in HUVECs. We observed that treatment with LPA $(5 \mu \mathrm{M})$ significantly enhanced IL- $1 \beta$ protein expression in HUVECs. In addition, LPA-induced IL- $1 \beta$ protein expression in HUVECs was blocked by Ki16425, AG1478, GM6001, and AF12198. A Rac inhibitor (toxin B) showed no suppressive effects (Figure 2). These results indicate that the enhancement of IL- $1 \beta$ protein expression by LPA in HUVECs is mediated by LPA1/3-, EGFR transactivation-, MMP-, and IL-1Rdependent mechanisms. Our previous study indicated that
LPA induced MMP activity and EGFR transactivation [13, 23, 24]. GM6001, a broad-spectrum MMP inhibitor, is known to reduce LPA-induced EGFR transactivation. In this study, we showed that GM6001 and AG1478 could inhibit LPA-induced IL- $1 \beta$ expression. These results indicated that LPA-induced IL- $1 \beta$ expression may act through EGFR transactivation.

3.3. LPA-Stimulated VEGF-C Protein Expression Is Mediated by EGFR Transactivation-, MMP-, LPA1/3-, and IL$1 R$-Dependent Mechanisms in HUVECs. The present study showed that IL- $1 \beta$ plays an important role in LPA-induced lymphangiogenesis. Moreover, we demonstrated that VEGF$\mathrm{C}$ mediates LPA-induced lymphangiogenesis. Since both IL$1 \beta$ and VEGF-C mediate LPA-induced lymphangiogenesis, we next investigated whether LPA-stimulated VEGF-C protein expression is mediated by an IL-1 receptor mechanism. LPA-induced VEGF-C protein expression in HUVECs was blocked by Ki16425, AG1478, GM6001, and AF12198. Pretreatment with a Rac inhibitor (toxin B) showed no suppressive effects (Figure 3 ). These results indicate that the enhancement of VEGF-C protein expression by LPA in HUVECs is also mediated through these pathways.

3.4. LPA-Stimulated Endothelial Cell Tube Formation In Vitro Is Mediated by EGFR Transactivation-, MMP-, LPA1/3-, and IL-1R-Dependent Mechanisms. To further verify whether LPA1/3, EGFR transactivation, and IL- $1 \beta$ play critical roles in LPA-induced endothelial cell tube formation, HUVECs 


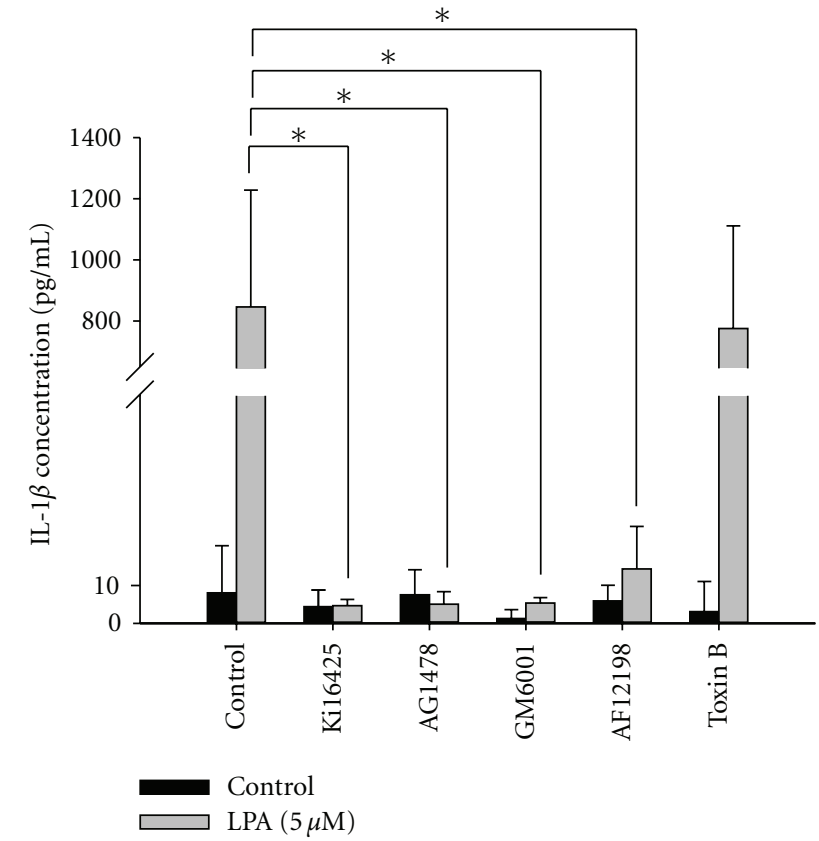

FIGURE 2: Lysophosphatidic acid- (LPA-) induced interleukin- (IL-) $1 \beta$ protein expression is mediated through endothelial growth factor receptor (EGFR) transactivation-, matrix metalloproteinase(MMP-), LPA1/3-, and IL-1R-dependent mechanisms in human umbilical vein endothelial cells (HUVECs). Starved HUVECs were pretreated with AG1478 (100 nM), AF12198 (10 nM), GM6001 $(10 \mu \mathrm{M}), \mathrm{Ki1} 6425(10 \mu \mathrm{M})$, and toxin B $(10 \mathrm{nM})$ for $1 \mathrm{~h}$, followed by LPA $(5 \mu \mathrm{M})$ treatment for an additional $24 \mathrm{~h}$. Conditioned medium was measured by an ELISA as described in "Methods". All ELISA data are expressed as the mean \pm SD from at least three independent experiments. ${ }^{*} P<.05,{ }^{* *} P<.01$, and ${ }^{* * *} P<.001$ indicate a statistically significant difference with the untreated control.

were pretreated with $100 \mathrm{nM}$ of AG1478, $10 \mathrm{nM}$ of AF12198, $10 \mu \mathrm{M}$ of GM6001, $10 \mu \mathrm{M}$ of Kil6425, or $10 \mathrm{nM}$ of toxin B for $1 \mathrm{~h}$, followed by LPA $(5 \mu \mathrm{M})$ treatment for an additional $24 \mathrm{~h}$, and then Matrigel tube formation assays were performed. After $6 \mathrm{~h}$ of plating, Matrigel of each experiment was fixed and subjected to an immunocytochemical assay. By immunostaining with PECAM-1, an endothelial cell marker, expression levels were stimulated by LPA induction and compared to the untreated control (Figure 4). These results further demonstrated that LPA-induced tube formation in HUVECs is mediated through LPA1/3-, EGFR transactivation-, and IL-1R-dependent mechanisms. According to immunostaining with Prox-1, we observed that LPA upregulated the expression of Prox-1, a lymphatic marker. These enhancement effects were also suppressed by an inhibitor of LPA1/3, EGFR kinase, a broad-spectrum MMP, and IL-1R. However, an inhibitor of Rac (toxin B) showed no suppressive effects (Figure 4). These results demonstrate that LPA-induced tube formation and lymphatic marker expressions are also mediated by LPA1/3-, EGFR transactivation-, and IL-1R-dependent mechanisms.

A previous study indicated that basal mRNA expression levels of lymphatic markers are relatively low in HUVECs

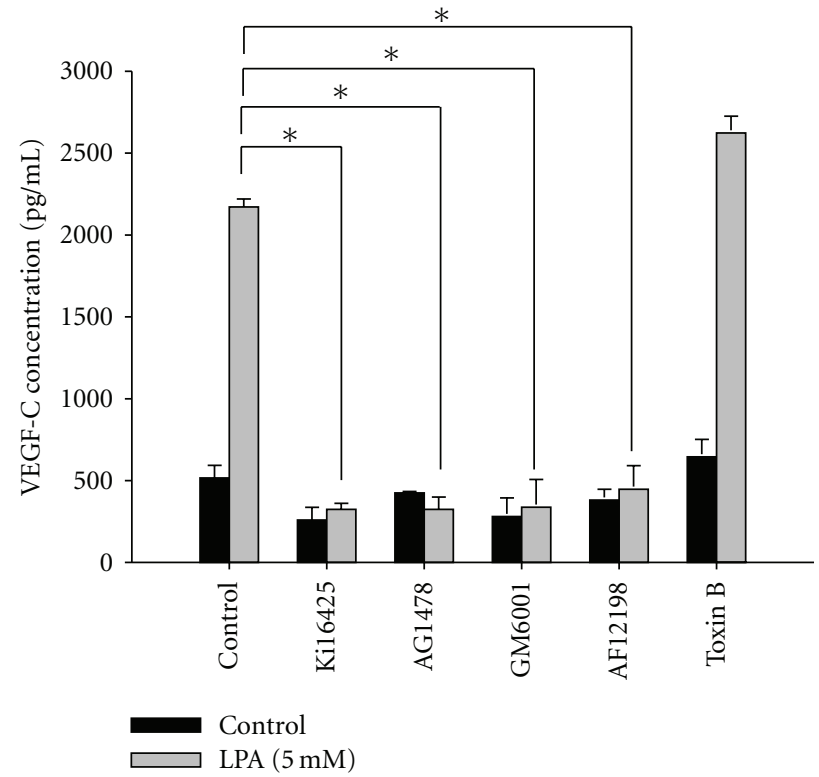

FIGURE 3: Lysophosphatidic acid- (LPA-) stimulated vascular endothelial growth factor- (VEGF-) C protein expression is mediated through endothelial growth factor receptor (EGFR) transactivation-, matrix metalloproteinase- (MMP-), LPA1/3-, and interleukin- (IL-) 1R-dependent mechanisms in human umbilical vein endothelial cells (HUVECs). Starved HUVECs were pretreated with AG1478 (100 nM), AF12198 (10 nM), GM6001 (10 $\mu \mathrm{M})$, Kil6425 $(10 \mu \mathrm{M})$, and toxin B $(10 \mathrm{nM})$ for $1 \mathrm{~h}$, followed by LPA $(5 \mu \mathrm{M})$ treatment for an additional $24 \mathrm{~h}$. Conditioned medium was measured by an ELISA as described in "Methods". All ELISA data are expressed as the mean \pm SD from at least three independent experiments. ${ }^{*} P<.05,{ }^{* *} P<.01$, and ${ }^{* * *} P<.001$ indicate a statistically significant difference with the untreated control.

[29]. In addition, IL-3 induces the transformation of blood endothelial cells (BECs) into lymphatic endothelial cells (LECs). In this study, we also reported that LPA induced lymphatic marker expressions in HUVECs. These findings suggested that non-LECs can be used to study lymphangiogenesis. However, the effects of LPA on adult LECs or endothelial stem cells will be important experiments to carry out.

Both inflammation and lymphangiogenesis are aggravated by enhanced production of cytokines, chemokines, growth factors, and lipid mediators. It was reported that expressions of LPA-induced inflammatory response genes are mediated through a nuclear factor- (NF-) $\kappa \mathrm{B}-$ dependent pathway [30]. Moreover, our data showed that LPA induces lymphangiogenesis via an IL- $1 \beta$-dependent pathway. Accordingly, LPA acts as a regulator of both lymphangiogenesis and inflammation. Like LPA, S1P is known to be a proinflammatory agent in the airway epithelium. In addition, S1P also plays a role in regulating lymphatic vessel maturation [31]. S1P1 is expressed in lymphocytes, and $\mathrm{S} 1 \mathrm{P}$ regulates migration of T-cells from nonlymphoid tissues to the lymph [system?] [32]. Moreover, human lymphatic ECs (HLECs) express S1P1 and S1P3, and S1P acts as a 

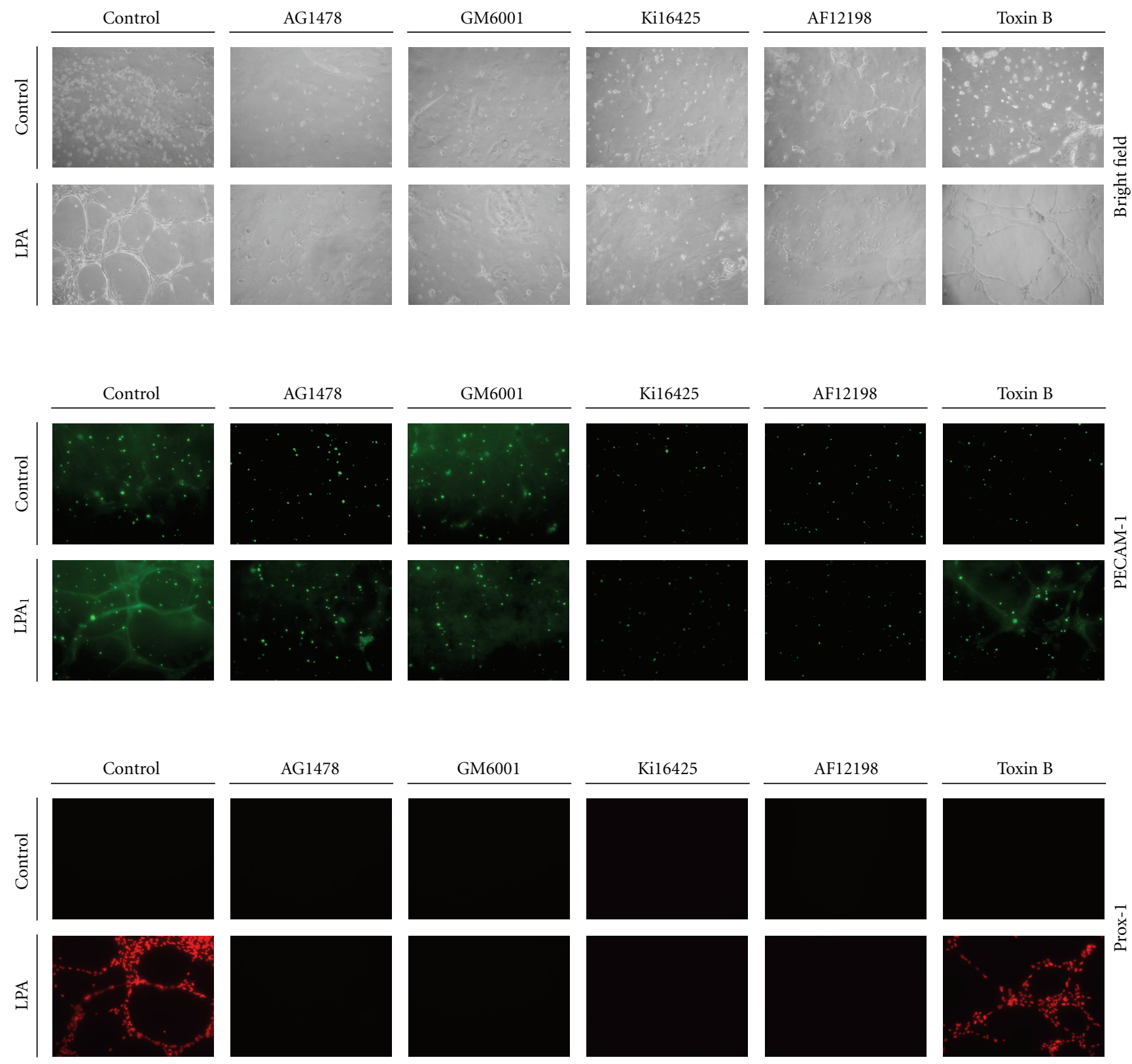

FIGURE 4: Lysophosphatidic acid- (LPA-) stimulated endothelial cell tube formation in vitro is mediated through endothelial growth factor receptor (EGFR) transactivation-, matrix metalloproteinase- (MMP-), LPA1/3-, and interleukin- (IL-) 1R-dependent mechanisms. Starved HUVECs were pretreated with AG1478 (100 nM), AF12198 (10 nM), GM6001 (10 $\mu \mathrm{M})$, Ki16425 $(10 \mu \mathrm{M})$, and toxin B $(10 \mathrm{nM})$ for $1 \mathrm{~h}$, followed by LPA $(5 \mu \mathrm{M})$ treatment for an additional $24 \mathrm{~h}$, and then cells were seeded onto Matrigel-coated plates. After $6 \mathrm{~h}$ of plating, Matrigel of each experiment was fixed and subjected to an immunocytochemical assay. The Matrigel was stained with PECAM-1 or a Prox-1 primary antibody followed by an FITC-conjugated secondary antibody. Images were taken after plating and visualized by immunofluorescence microscopy.

lymphatic regulator [25]. These results further confirm that bioactive lipids may be regulators of both inflammation and lymphangiogenesis.

The lymphatic vascular system has critical roles in regulating interstitial pressure, homeostasis, and inflammation. Several studies suggested that new lymphatic vessel formation can be caused by chronic inflammation. For example, TNF- $\alpha$, a proinflammatory factor, was found to be a mediator driving blood vessel remodeling and lymphangiogenesis in inflammation [33]. VEGF-C is mainly derived from inflammatory cells, which are involved in regulating lymphangiogenesis via VEGFR-3 signaling in LECs [34]. Moreover, Prox- 1 and podoplanin were shown to be induced by inflammatory cytokines in differentiated ECs [29]. Those results suggest that inflammation evokes the formation of new lymphatic vessels. 


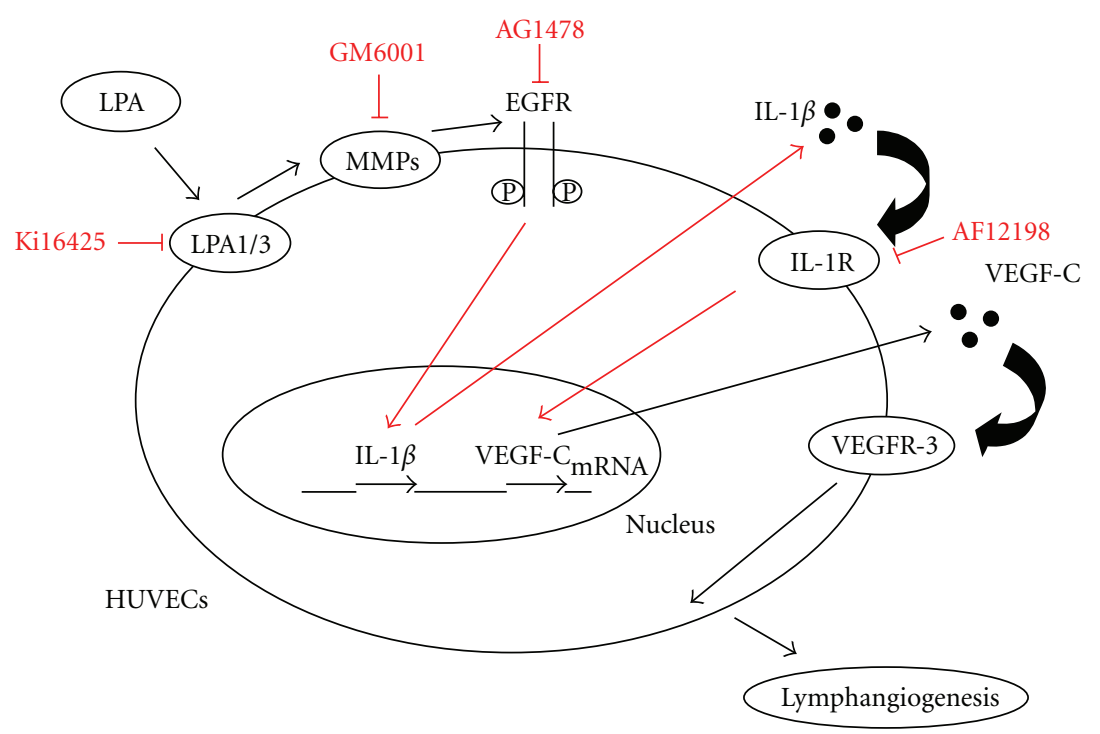

FIGURE 5: Proposed mechanisms of lysophosphatidic acid- (LPA-) induced lymphangiogenesis in human endothelial cells.

Cytokines, such as TNF- $\alpha$ and IL- $1 \beta$, were reported to enhance VEGF-C expression in an NF- $\kappa$ B-dependent manner in HUVECs $[26,33]$. In addition, IL- $1 \beta$ also induced lymphangiogenesis in mouse corneas through upregulating the production of VEGF-C and VEGF-D [27]. Our previous study demonstrated that LPA induced VEGF-C expression and lymphangiogenesis in human ECs. Furthermore, LPA stimulated IL- 8 and MCP-1 expressions via an IL-1dependent pathway [28]. In this study, we clarified that the LPA-induced lymphangiogenesis cascade acts through an IL$1 \beta$-dependent pathway which is a crucial pathway involved in the inflammatory response. This is consistent with a previous study demonstrating that IL- $1 \beta$ plays a key role in lymphangiogenesis [27].

As shown in Figure 2, we found LPA-induced IL-1 $\beta$ protein expression in human ECs. However, the enhancement effects were abolished by treatment with AF12198, an inhibitor of the IL-1 receptor, suggesting that the regulation of IL-1 has a positive-feedback mechanism. Our results are consistent with those of the Dinarello and Savage (1989) study showing that IL-1 has a self-augmentation induction mechanism, which allows a positive-feedback mechanism to amplify the effects of cytokines within the local environment [35].

In summary, this study is the first to establish that IL- $1 \beta$ is necessary for LPA-induced VEGF-C expression and lymphangiogenesis in human endothelial cells, which suggests that both LPA and IL- $1 \beta$ may be potent targets for generating therapeutics against lymphangiogenesis and tumor metastasis.

\section{Conclusions}

Our data clearly indicate that LPA-induced VEGF-C expression is largely mediated by the IL-1 receptor. Furthermore, LPA stimulated lymphatic marker expressions through upregulating IL- $1 \beta$ protein expression in HUVECs, thereby inducing the progression of lymphangiogenesis (Figure 5). These findings suggest that LPA plays a critical role in inflammation-induced lymphangiogenesis.

\section{Acknowledgments}

The authors thank Dr. S. U. Chen at National Taiwan University Hospital for providing umbilical cords for the endothelial cells preparation and Chi Iou Lin for technical assistance.

\section{References}

[1] E. J. Goetzl and S. An, "Diversity of cellular receptors and functions for the lysophospholipid growth factors lysophosphatidic acid and sphingosine 1-phosphate," FASEB Journal, vol. 12, no. 15, pp. 1589-1598, 1998.

[2] W. H. Moolenaar, "Bioactive lysophospholipids and their G protein-coupled receptors," Experimental Cell Research, vol. 253, no. 1, pp. 230-238, 1999.

[3] A. M. Spiegel, "G protein defects in signal transduction," Hormone Research, vol. 53, no. 3, pp. 17-22, 2000.

[4] Y. Yatomi, S. Yamamura, F. Ruan, S. Kume, Y. Ozaki, and Y. Igarashi, "N,N-dimethylsphingosine 1-phosphate activates human platelets," FEBS Letters, vol. 417, no. 3, pp. 341-344, 1997.

[5] T. Eichholtz, K. Jalink, I. Fahrenfort, and W. H. Moolenaar, "The bioactive phospholipid lysophosphatidic acid is released from activated platelets," Biochemical Journal, vol. 291, no. 3, pp. 677-680, 1993.

[6] Y. Xu, Z. Shen, D. W. Wiper et al., "Lysophosphatidic acid as a potential biomarker for ovarian and other gynecologic cancers," Journal of the American Medical Association, vol. 280, no. 8, pp. 719-723, 1998.

[7] G. A. Piazza, J. L. Ritter, and C. A. Baracka, "Lysophosphatidic acid induction of transforming growth factors $\alpha$ and $\beta$ : modulation of proliferation and differentiation in cultured 
human keratinocytes and mouse skin," Experimental Cell Research, vol. 216, no. 1, pp. 51-64, 1995.

[8] T. S. Panetti, H. Chen, T. M. Misenheimer, S. B. Getzler, and D. F. Mosher, "Endothelial cell mitogenesis induced by LPA: inhibition by thrombospondin-1 and thrombospondin2," Journal of Laboratory and Clinical Medicine, vol. 129, no. 2, pp. 208-216, 1997.

[9] C. Rizza, N. Leitinger, J. Yue et al., "Lysophosphatidic acid as a regulator of endothelial/leukocyte interaction," Laboratory Investigation, vol. 79, no. 10, pp. 1227-1235, 1999.

[10] P. Xia, L. Wang, J. R. Gamble, and M. A. Vadas, "Activation of sphingosine kinase by tumor necrosis factor- $\alpha$ inhibits apoptosis in human endothelial cells," The Journal of Biological Chemistry, vol. 274, no. 48, pp. 34499-34505, 1999.

[11] H. Lee, E. J. Goetzl, and S. An, "Lysophosphatidic acid and sphingosine 1-phosphate stimulate endothelial cell wound healing," American Journal of Physiology, vol. 278, no. 3, pp. C612-C618, 2000.

[12] M. A. Watsky, M. Griffith, D.-A. Wang, and G. J. Tigyi, "Phospholipid growth factors and corneal wound wealing," Annals of the New York Academy of Sciences, vol. 905, pp. 142$158,2000$.

[13] W. T. Wu, C.-N. Chen, C. I. Lin, J. H. Chen, and H. Lee, "Lysophospholipids enhance matrix metalloproteinase2 expression in human endothelial cells," Endocrinology, vol. 146, no. 8, pp. 3387-3400, 2005.

[14] S. Kato, H. Shimoda, R.-C. Ji, and M. Miura, "Lymphangiogenesis and expression of specific molecules as lymphatic endothelial cell markers," Anatomical Science International, vol. 81, no. 2, pp. 71-83, 2006.

[15] R.-C. Ji, "Lymphatic endothelial cells, lymphangiogenesis, and extracellular matrix," Lymphatic Research and Biology, vol. 4, no. 2, pp. 83-100, 2006.

[16] R.-C. Ji, "Lymphatic endothelial cells, tumor lymphangiogenesis and metastasis: new insights into intratumoral and peritumoral lymphatics," Cancer and Metastasis Reviews, vol. 25, no. 4, pp. 677-694, 2006.

[17] T. L. Underiner, B. Ruggeri, and D. E. Gingrich, "Development of vascular endothelial growth factor receptor (VEGFR) kinase inhibitors as anti-angiogenic agents in cancer therapy," Current Medicinal Chemistry, vol. 11, no. 6, pp. 731-745, 2004.

[18] V. Joukov, K. Pajusola, A. Kaipainen et al., "A novel vascular endothelial growth factor, VEGF-C, is a ligand for the Flt4 (VEGFR-3) and KDR (VEGFR-2) receptor tyrosine kinases," EMBO Journal, vol. 15, no. 2, pp. 290-298, 1996.

[19] V. Joukov, T. Sorsa, V. Kumar et al., "Proteolytic processing regulates receptor specificity and activity of VEGF-C," EMBO Journal, vol. 16, no. 13, pp. 3898-3911, 1997.

[20] M. Jeltsch, A. Kaipainen, V. Joukov et al., "Hyperplasia of lymphatic vessels in VEGF-C transgenic mice," Science, vol. 276, no. 5317, pp. 1423-1425, 1997.

[21] S.-J. Oh, M. M. Jeltsch, R. Birkenhäger et al., "VEGF and VEGF-C: specific induction of angiogenesis and lymphangiogenesis in the differentiated avian chorioallantoic membrane," Developmental Biology, vol. 188, no. 1, pp. 96-109, 1997.

[22] J.-L. Su, J.-Y. Shih, M.-L. Yen et al., "Cyclooxygenase-2 induces EP1- and HER-2/Neu-dependent vascular endothelial growth factor-C up-regulation: a novel-mechanism of lymphangiogenesis in lung adenocarcinoma," Cancer Research, vol. 64, no. 2, pp. 554-564, 2004.
[23] C.-I. Lin, C.-N. Chen, M.-T. Huang et al., "Lysophosphatidic acid upregulates vascular endothelial growth factor-C and tube formation in human endothelial cells through LPA1/3, COX-2, and NF- $\kappa$ B activation- and EGFR transactivationdependent mechanisms," Cellular Signalling, vol. 20, no. 10, pp. 1804-1814, 2008.

[24] C.-I. Lin, C.-N. Chen, M.-T. Huang et al., "Lysophosphatidic acid up-regulates vascular endothelial growth factor- $\mathrm{C}$ and lymphatic marker expressions in human endothelial cells," Cellular and Molecular Life Sciences, vol. 65, no. 17, pp. 27402751, 2008.

[25] C. M. Yoon, B. S. Hong, H. G. Moon et al., "Sphingosine1-phosphate promotes lymphangiogenesis by stimulating S1P1/Gi/PLC/Ca ${ }^{2+}$ signaling pathways," Blood, vol. 112, no. 4, pp. 1129-1138, 2008.

[26] A. Ristimäki, K. Narko, B. Enholm, V. Joukov, and K. Alitalo, "Proinflammatory cytokines regulate expression of the lymphatic endothelial mitogen vascular endothelial growth factor-C," The Journal of Biological Chemistry, vol. 273, no. 14, pp. 8413-8418, 1998.

[27] K. Watari, S. Nakao, A. Fotovati et al., "Role of macrophages in inflammatory lymphangiogenesis: enhanced production of vascular endothelial growth factor $\mathrm{C}$ and $\mathrm{D}$ through NF- $\kappa \mathrm{B}$ activation," Biochemical and Biophysical Research Communications, vol. 377, no. 3, pp. 826-831, 2008.

[28] C. I. Lin, C.-N. Chen, J. H. Chen, and H. Lee, "Lysophospholipids increase IL-8 and MCP-1 expressions in human umbilical cord vein endothelial cells through an IL-1 dependent mechanism," Journal of Cellular Biochemistry, vol. 99, no. 4, pp. 1216-1232, 2006.

[29] M. Gröger, R. Loewe, W. Holnthoner et al., "IL-3 induces expression of lymphatic markers Prox-1 and podoplanin in human endothelial cells," Journal of Immunology, vol. 173, no. 12, pp. 7161-7169, 2004.

[30] S.-U. Chen, C.-H. Chou, H. Lee, C.-N. Ho, C.-W. Lin, and Y.-S. Yang, "Lysophosphatidic acid up-regulates expression of interleukin-8 and -6 in granulosa-lutein cells through its receptors and nuclear factor- $\kappa \mathrm{B}$ dependent pathways: implications for angiogenesis of corpus luteum and ovarian hyperstimulation syndrome," Journal of Clinical Endocrinology and Metabolism, vol. 93, no. 3, pp. 935-943, 2008.

[31] T. H. M. Pham, P. Baluk, Y. Xu et al., "Lymphatic endothelial cell sphingosine kinase activity is required for lymphocyte egress and lymphatic patterning," Journal of Experimental Medicine, vol. 207, no. 1, pp. 17-27, 2010.

[32] L. G. Ledgerwood, G. Lal, N. Zhang et al., "The sphingosine 1-phosphate receptor 1 causes tissue retention by inhibiting the entry of peripheral tissue $\mathrm{T}$ lymphocytes into afferent lymphatics," Nature Immunology, vol. 9, no. 1, pp. 42-53, 2008.

[33] P. Baluk, L.-C. Yao, J. Feng et al., "TNF- $\alpha$ drives remodeling of blood vessels and lymphatics in sustained airway inflammation in mice," Journal of Clinical Investigation, vol. 119, no. 10, pp. 2954-2964, 2009.

[34] P. Baluk, T. Tammela, E. Ator et al., "Pathogenesis of persistent lymphatic vessel hyperplasia in chronic airway inflammation," Journal of Clinical Investigation, vol. 115, no. 2, pp. 247-257, 2005.

[35] C. A. Dinarello and N. Savage, "Interleukin-1 and its receptor," Critical Reviews in Immunology, vol. 9, no. 1, pp. 1-20, 1989. 


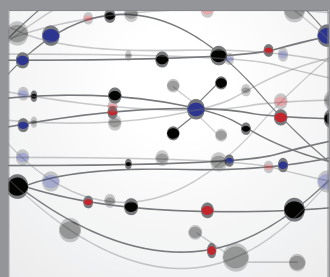

The Scientific World Journal
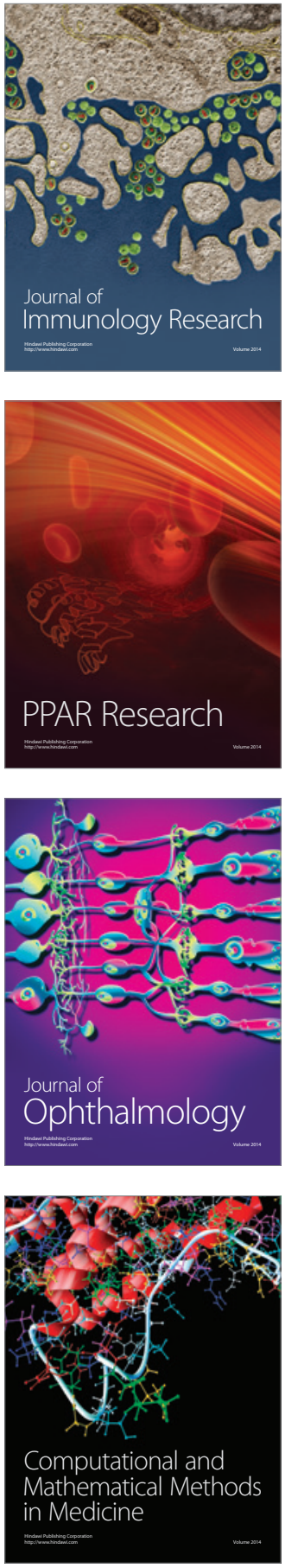

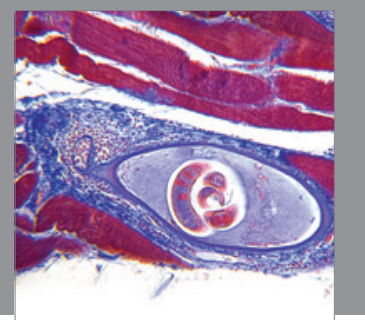

Gastroenterology

Research and Practice
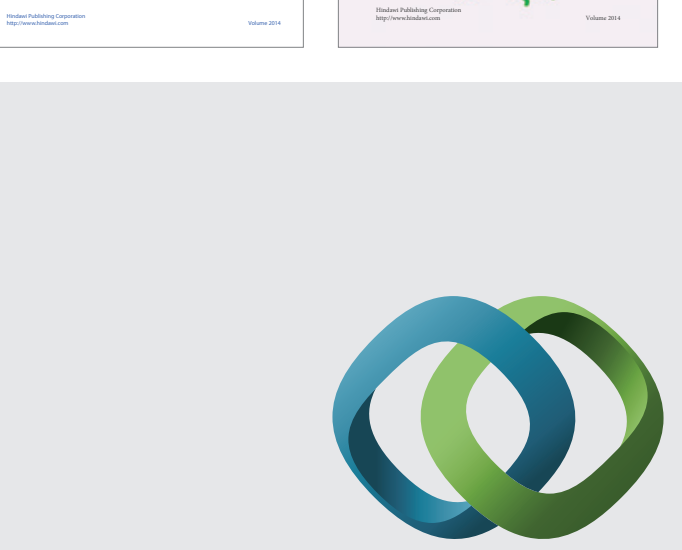

\section{Hindawi}

Submit your manuscripts at

http://www.hindawi.com
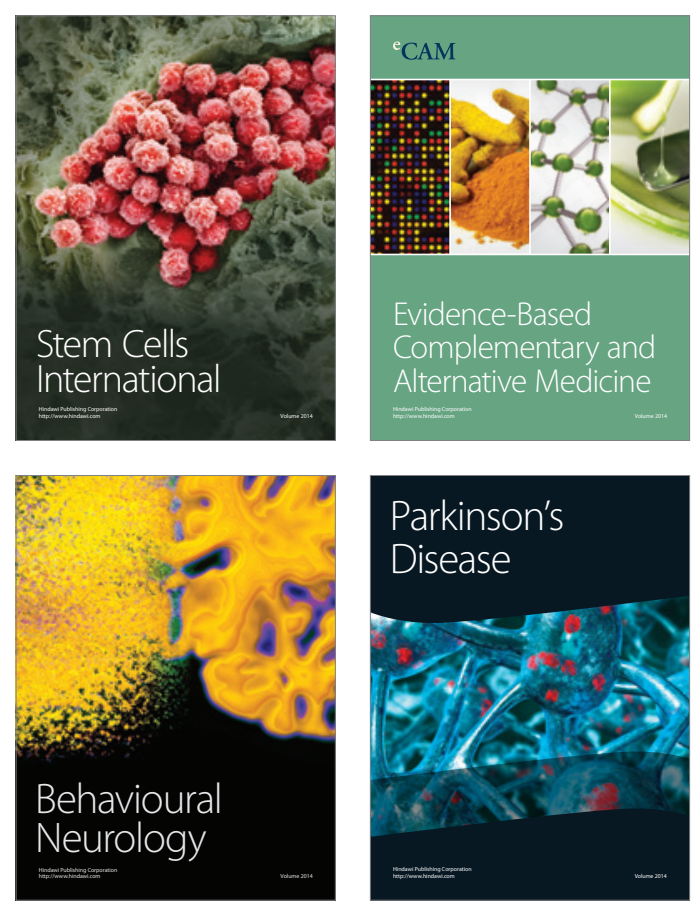

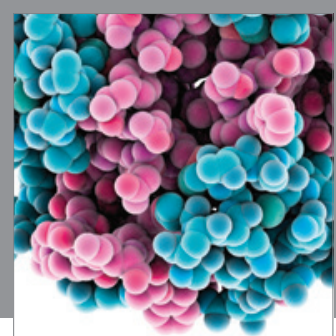

Journal of
Diabetes Research

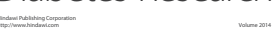

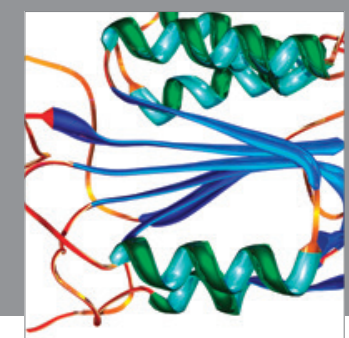

Disease Markers
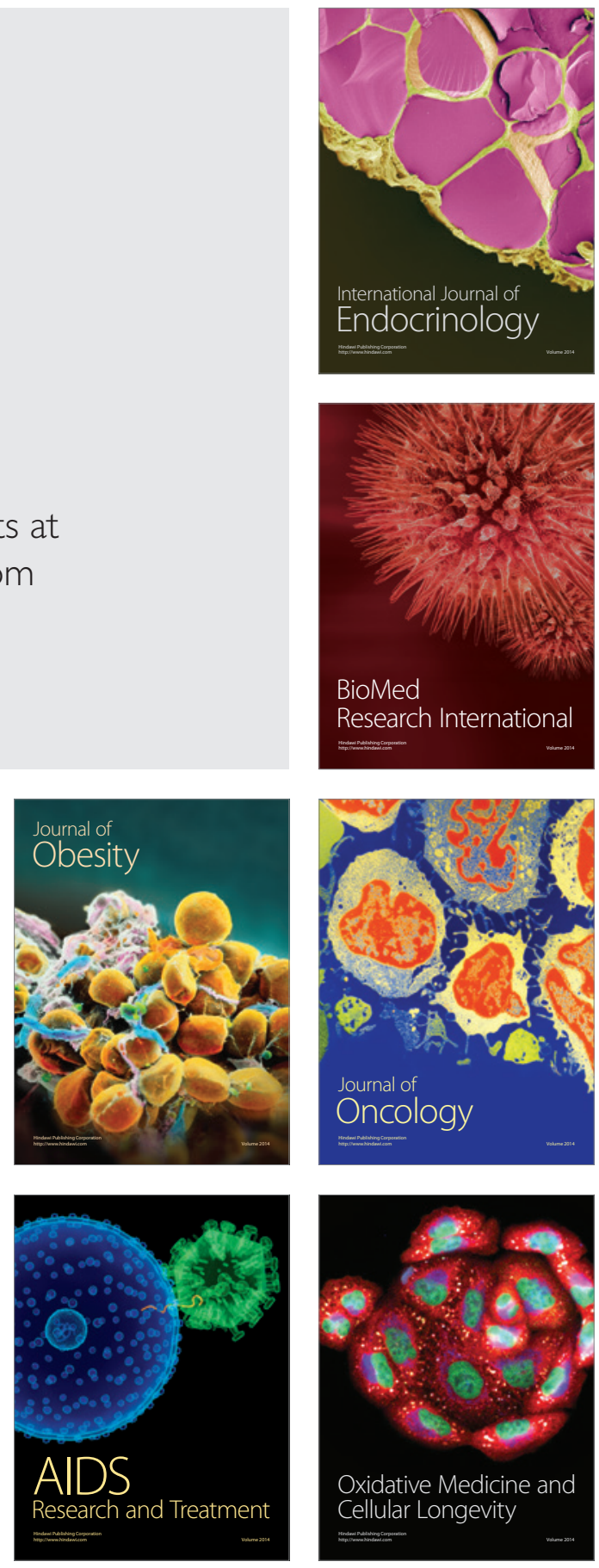